\title{
Electronic and atomic shell structure in aluminum nanowires
}

\author{
A. I. Mares ${ }^{1}$, D. F. Urban ${ }^{2}$, J. Bürki ${ }^{3}$, H. Grabert ${ }^{2}$, \\ C. A. Stafford ${ }^{3}$ and J. M. van Ruitenbeek ${ }^{1}$ \\ ${ }^{1}$ Kamerlingh Onnes Laboratorium, Universiteit Leiden, P.O. Box 9504, 2300 RA \\ Leiden, The Netherlands \\ ${ }^{2}$ Physikalisches Institut, Albert-Ludwigs-Universität, D-79104 Freiburg, \\ Germany \\ ${ }^{3}$ Department of Physics, University of Arizona, Tucson, Arizona 85721, USA \\ E-mail: urban@physik.uni-freiburg.de
}

Submitted to: Nanotechnology , 14 July 2018

\begin{abstract}
.
We report experiments on aluminum nanowires in ultra-high vacuum at room temperature that reveal a periodic spectrum of exceptionally stable structures. Two "magic" series of stable structures are observed: At low conductance, the formation of stable nanowires is governed by electronic shell effects whereas for larger contacts atomic packing dominates. The crossover between the two regimes is found to be smooth. A detailed comparison of the experimental results to a theoretical stability analysis indicates that while the main features of the observed electron-shell structure are similar to those of alkali and noble metals, a sequence of extremely stable wires plays a unique role in Aluminum. This series appears isolated in conductance histograms and can be attributed to "superdeformed" non-axisymmetric nanowires.
\end{abstract}

PACS numbers: 73.40.Jn, 61.46.+w, 68.65.La 


\section{Introduction}

Metal nanowires have attracted considerable interest in the past decade, experimental research has burgeoned, and various techniques have been employed to produce them for many different materials (see [1 for a recent review). Conductance histograms, built out of thousands of conductance traces recorded during the breaking of a nanowire in a mechanically controllable break junction (MCBJ), reveal the existence of exceptionally stable wires: Due to their stability, these wires are formed more often than others, and show up as peaks in a conductance histogram taken at a temperature large enough to allow the contact to explore the available configuration space.

Such experiments on alkali metals 2, 3, 4, have revealed a sequence of stable "magic" wires that is periodic in $\sqrt{G}$, where $G$ is the electrical conductance. A theoretical analysis of nanowire stability [5, 6, 7, within the nanoscale free-electron model (NFEM) [8, 9, 10, has connected this magic sequence of wires to the electron shell structure, similar to what is known for metal clusters [11, 12, 13. Groups of discrete transverse electronic states are bunched together, with gaps between them. When the electronic Fermi energy is within one of these gaps, the wire is exceptionally stable. The periodicity in $\sqrt{G}$ can be understood semi-classically: writing the mesoscopic contribution to the wire energy as a series in classical periodic orbits in a circular billiard [2, 5, 10, the main contribution is found to come from the diameter orbit, and to be periodic in the wire radius, and thus proportional to $\sqrt{G}$ via the Sharvin formula [14, 15]. A supershell structure was also observed [16] in the form of a periodic modulation of the peak heights, and it was argued that the stable wires at the nodes of the supershell structure are non-axisymmetric [6, 17, 18,

At larger wire diameters, and therefore larger conductance, a crossover to an atomic shell structure was found [19. In this case, shell closings correspond to crystalline wires with the completion of additional atomic layers. Recently, electronshell structure has also been observed for the noble metals gold [20, 21], silver [22, and copper 22]. Except for the latter, the crossover to atomic shell effects at larger diameter could be confirmed [21, 22].

Inspired by these results and by the research done on metal clusters, we extend our investigation to multivalent metals, choosing as a first step aluminum.

Conductance histograms for aluminum atomic size contacts recorded using the MCBJ technique at $4.2 \mathrm{~K}$ were first reported by Yanson et al 23. Four peaks were identified situated close to $1,2,3$ and 4 times the conductance quantum $G_{0}=2 e^{2} / \mathrm{h}$. Scheer et al 24] analyzed the transmission modes for $\mathrm{Al}$ point contacts by recording current-voltage characteristics in the superconducting state. For a single atom, three modes were found to contribute to the conductance, with individual transmissions lower than one summing together to a total conductance of about one quantum unit.

A previous experiment on aluminum shell structure 25] reports both experimental results and molecular dynamics simulations of the evolution of $\mathrm{Al}$ nanowires while breaking. The experiment was performed using a scanning tunnelling microscope (STM) at room temperature (RT) in ultra high vacuum (UHV). The authors attribute the oscillation period of the peaks to filling of octagonal facets, claiming that the electronic shell effect is absent. Our results reported below are significantly different since we present evidence of electronic shell effects. The difference with our experiments appear in several aspects: Firstly, in the histogram from 25 there is a large contribution in the low conductance regime, including below $1 G_{0}$. This is usually an indication of the presence of contaminants, especially expected in the 
case of STM soft indentations [26. Secondly, in 25] the peaks have low weight and a derivative is performed in order to identify their position. Moreover, from one single histogram one cannot exclude the occurrence of electronic shell effects, since electronic and atomic shell effects compete in the stabilization of the nanowire and in different measurements one or the other effect can dominate, as it will be shown below. Although the experimental results in [25] agree with the theoretical predictions based on molecular dynamics simulations, the latter calculation does not take into account the electronic contribution to the total energy of the nanowire, excluding the electronic shell effects from consideration.

This paper is organized as follows. The experimental results are presented in section 2, followed by a detailed analysis of the data in section 3. A theory explaining the observed electronic shell structure and allowing the prediction of the shapes of the wires is presented in section 3.1. Atomic shells and the crossover between electronic and atomic shell effects are analyzed in section 3.2. Section 3.3 addresses the issue of superdeformed nanowires. Finally, a short summary is given in section 4 .

\section{Experimental results}

We report conductance histograms recorded for $\mathrm{Al}$ nanowires in UHV at room temperature using the MCBJ set-up presented in 21] and 22]. A metallic nanowire is bent in a controlled way and its diameter at the weakest point is reduced down to atomic size. The wire is initially broken under UHV, ensuring in this way clean surfaces. In order to perform a reliable statistical analysis we have investigated six different samples. The behavior was reproduced on every sample and typical histograms are shown in figures 1, 2, 3, In the histogram from figure 1 we can see a remarkably long series of peaks up to a conductance value of $100 G_{0}$.

The first step in finding the origin of the peaks in the conductance histogram is to verify whether the peaks show a certain periodicity. We can see in figure 1(b) and (c) that a linear dependence is found when plotting the square root of the conductance as a function of the peak index, while when plotting directly the conductance the dependence is far from linear. Note that the square root of the conductance is proportional to the root-mean-square (rms) radius $\rho$ of the wire, as can be inferred from the corrected Sharvin formula [14, 15],

$$
G_{S}=G_{0}\left(\frac{k_{F}^{2} \rho^{2}}{4}-\frac{k_{F} \mathcal{P}}{4 \pi}+\frac{1}{6}\right)
$$

which gives a semi-classical approximation to the conductance. Here $\mathcal{P}$ is the perimeter of the wire's cross section and $k_{F}$ is the Fermi wavevector. In the case of the histogram of figure 1 the period found is $\Delta \sqrt{G / G_{0}}=0.29 \pm 0.002$.

As for the other metals, we have studied how the histograms vary from one experiment to the next, and a characteristically different histogram can be seen in figure 2. There a crossover to a different period occurs. Two periods of oscillation can be identified around $\Delta \sqrt{G / G_{0}}=0.47$ and 0.25 , with a crossover at a conductance value of about $40 G_{0}$. The first period is similar to the period of the electronic shell effect in noble metals [21, 22]. The second one, similar to the period of the histogram of figure 1 is somewhat larger than what was obtained for the atomic shell effect in noble metals, for which typically $\Delta \sqrt{G / G_{0}}$ is found to be around 0.21 .

Sometimes a new type of histogram appears having only two or three peaks as one can see in figure 3. This type of histogram appears at the beginning of the 
measurements on a fresh sample just after the initial breaking, and we see it in 7 out of 30 histograms. The position of the two major peaks is always close to 14 and 22 $G_{0}$. In two of these histograms a peak near $5 G_{0}$ also appears. All three peaks are related to very long plateaus at these values in the individual conductance traces [see inset of figure 3 .

Finally, in figure 4 we show a conductance histogram recorded for aluminum in air at room temperature. We see distinct peaks that have a period close to the electronic shell effect found in noble metals and for $\mathrm{Al}$ in $\mathrm{UHV}$. It is known that bulk $\mathrm{Al}$ is a very reactive metal that forms an oxide layer on the surface. Possibly this layer prevents the immediate contamination inside the bulk of the nanowire.

\section{Theoretical analysis of Al histograms}

In order to obtain more information about the stable wire configurations realized in experiments, we have analyzed in detail the conductance histogram of figure 2 We fit the data set with a function consisting of a sum of peaks of lorentzian shape with height, width and peak position as fitting parameters, and the result is shown
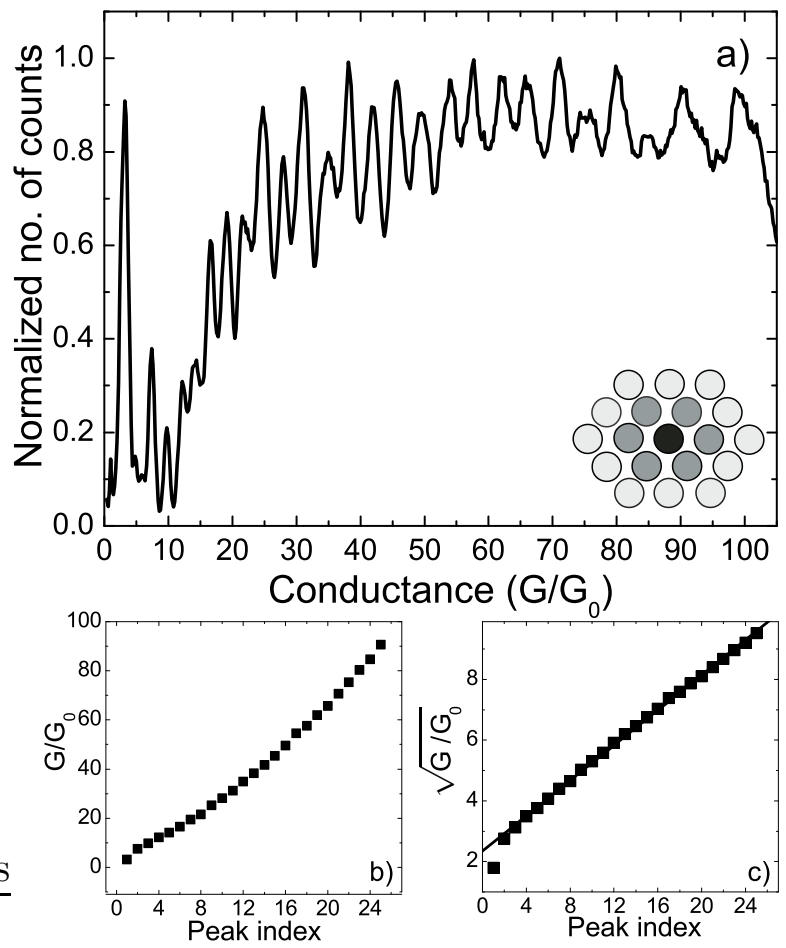

Figure 1. (a) Conductance histogram for aluminum at RT-UHV constructed from 7000 scans at a bias voltage of $50 \mathrm{mV}$. The inset shows the presumed atomic positions in the normal cross-section of the wire. In (b) we plot the conductance values at the peak position vs. their index while in (c) $\sqrt{G / G_{0}}$ for the peak positions vs. peak index. For (c) a good linear dependence is obtained with a period $\Delta \sqrt{G / G_{0}}=0.29 \pm 0.002$. 
in figure 5 and table 1. Note that the number of peaks used for the fit is not unique, as the fit can always be improved by including additional broad peaks of lower intensities. However, the parameters of additional peaks can not be determined with high confidence, especially at larger conductance where the curve is more noisy.

Besides the 18 dominant conductance peaks, marked with numbered black arrows

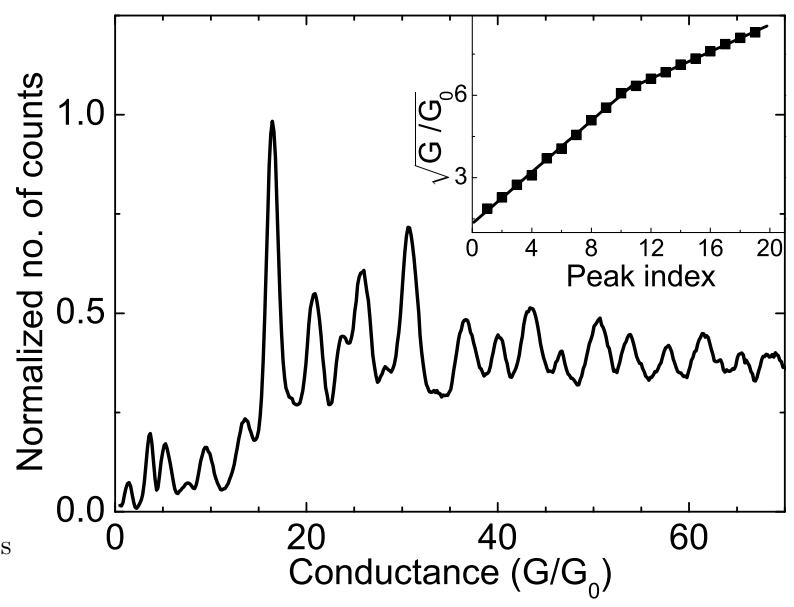

Figure 2. Conductance histogram for $\mathrm{Al}$ taken under RT-UHV conditions, recorded at a bias voltage of $20 \mathrm{mV}$ constructed from 2000 scans. Inset: the peak oscillation has two periods: in the low conductance regime up to about 40 $G_{0}$ the period is $\Delta \sqrt{G / G_{0}}=0.47 \pm 0.03$ whereas for larger diameters we find $\Delta \sqrt{G / G_{0}}=0.25 \pm 0.01$.

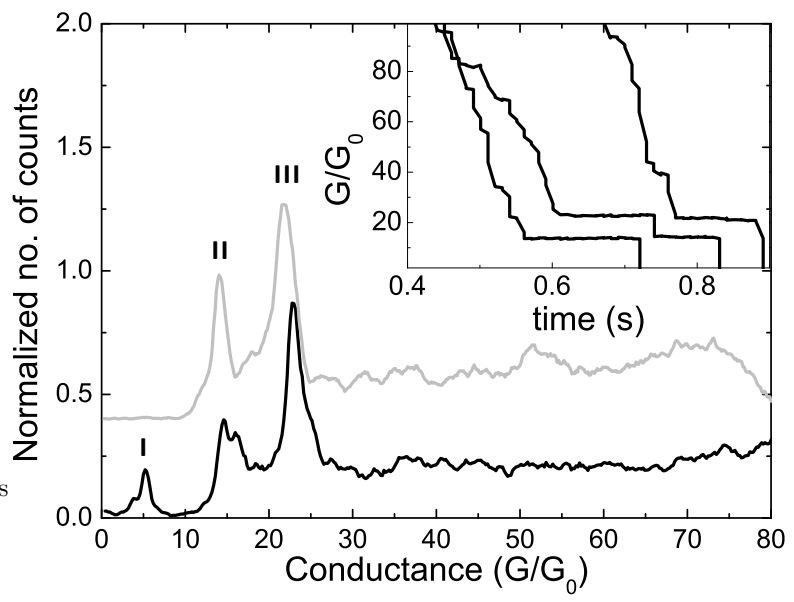

Figure 3. Special type of histograms recorded for Al at RT-UHV showing two dominant peaks near 14 and $22 G_{0}$, while the lower curve shows a third peak near $5 G_{0}$. The two curves are vertically offset for clarity. The histograms are recorded on two different samples, both at a bias voltage of $20 \mathrm{mV}$ containing 1000 traces each. In the individual conductance traces long plateaus can be seen at these values, shown in the inset. 


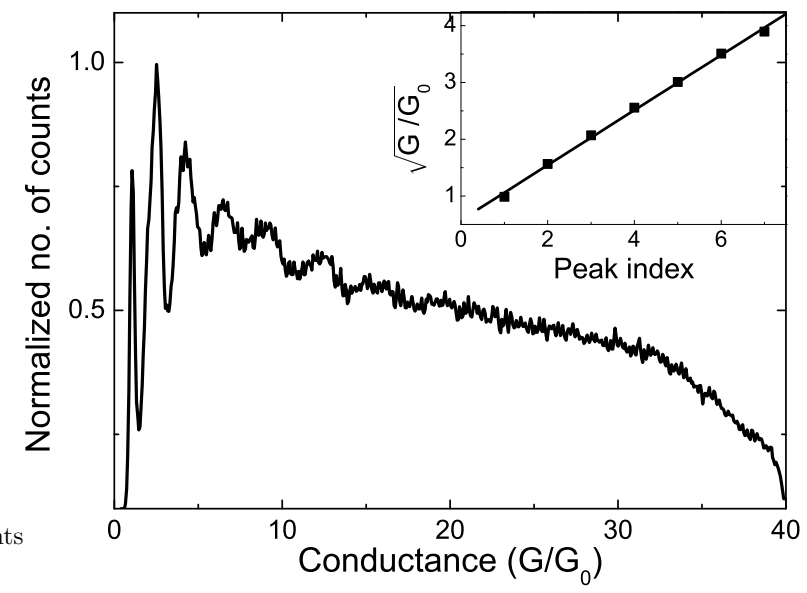

Figure 4. Conductance histogram for $\mathrm{Al}$ at room temperature in air constructed from 1000 traces at a bias voltage of $20 \mathrm{mV}$. Inset: the peak oscillation has a period $\Delta \sqrt{G / G_{0}}=0.48 \pm 0.04$.

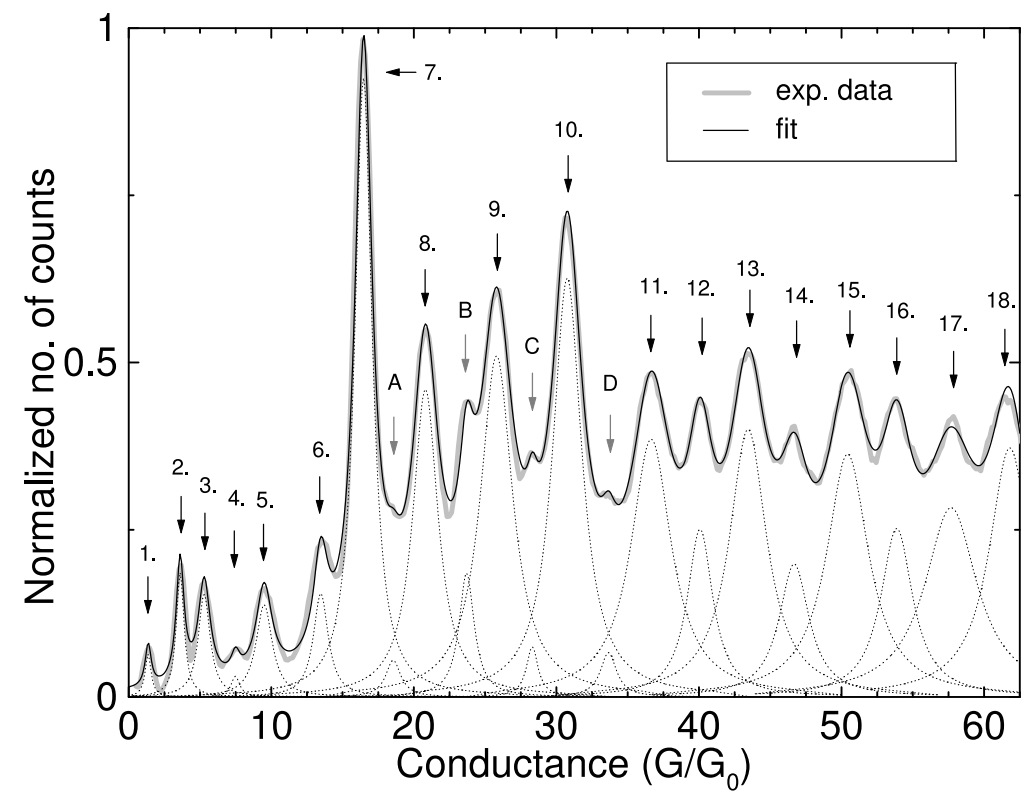

Figure 5. Experimental conductance histogram data from figure 2 fitted with lorentzian peaks. The dotted lines show the individual peaks. The positions, weights, and widths of the peaks are given in table 1

in figure 5. we can identify a number of very shallow peaks in the conductance histogram. They originate from less stable wire configurations, that are less frequently realized. These "peaks" are marked with capital letters A-D in figure 5 .

Figure 6] shows $\sqrt{G}$ versus peak number for three different peak-selection criteria: Peaks 1-18 of table 1 are plotted as red squares, reproducing the inset of figure 2 with 
Table 1. Conductance $G_{\exp }$, relative weight, and width $\Delta G$ of the peaks in the histogram shown in figure 5 . The values were obtained by fitting the data set to a sum of lorentzian peaks, with height, width, and peak position as fitting parameters. The next two columns give the parameters of the stable $\mathrm{Al}$ nanowires determined by a linear stability analysis within the NFEM: $G_{t h}$ is the quantized conductance and $\lambda$ is the quadrupole deformation [cf. (2)]. The last two columns give the mean channel transmission $\left\langle T_{n}\right\rangle \equiv G_{\text {exp }} / G_{t h}$ or series resistance $R_{s}$ needed to shift the theoretical peaks down to the corresponding experimental values. For completeness, the NFEM predictions are shown for peaks $1-5$, although the model is not expected to be quantitatively accurate for few-atom contacts in multivalent metals, as evidenced by the large values of the effective series resistance, or the small channel transmissions 24]. The last three lines of the Table give the positions of the three main peaks of figure 3 and the corresponding theoretical parameters.

\begin{tabular}{|c|c|c|c|c|c|c|c|}
\hline peak & $\begin{array}{c}G_{\exp } \\
{\left[G_{0}\right]}\end{array}$ & $\begin{array}{c}\text { weight } \\
{[\%]}\end{array}$ & $\begin{array}{l}\Delta G \\
{\left[G_{0}\right]}\end{array}$ & $\begin{array}{l}G_{t h} \\
{\left[G_{0}\right]}\end{array}$ & $\lambda$ & $\left\langle T_{n}\right\rangle$ & $\begin{array}{l}R_{s} \\
{[\Omega]}\end{array}$ \\
\hline 1 & 1.4 & 0.2 & 0.5 & 3 & 0 & 0.45 & 5185 \\
\hline 2 & 3.6 & 0.8 & 0.7 & 5 & 0.17 & 0.72 & 993 \\
\hline 3 & 5.3 & 1.1 & 1.0 & $6-7$ & $0-0.3$ & $0.76-0.88$ & $289-596$ \\
\hline 4 & 7.5 & 0.2 & 0.7 & 9 & 0.11 & 0.83 & 291 \\
\hline 5 & 9.5 & 1.3 & 1.4 & 12 & 0 & 0.79 & 283 \\
\hline 6 & 13.5 & 1.3 & 1.3 & 14 & 0.3 & 0.96 & 36 \\
\hline 7 & 16.5 & 9.2 & 1.5 & 17 & 0 & 0.97 & 24 \\
\hline A & 18.6 & 0.5 & 1.3 & - & - & - & - \\
\hline 8 & 20.8 & 6.7 & 2.2 & 23 & 0 & 0.90 & 59 \\
\hline B & 23.7 & 1.6 & 1.3 & - & - & - & - \\
\hline 9 & 25.8 & 9.5 & 2.8 & 29 & 0.06 & 0.89 & 55 \\
\hline $\mathrm{C}$ & 28.3 & 0.5 & 1.0 & - & - & - & - \\
\hline 10 & 30.8 & 10.5 & 2.5 & 34 & 0 & 0.90 & 40 \\
\hline D & 33.7 & 0.6 & 1.4 & - & - & - & - \\
\hline 11 & 36.7 & 9.0 & 3.4 & 42 & 0 & 0.87 & 45 \\
\hline 12 & 40.0 & 3.7 & 2.2 & - & - & - & - \\
\hline 13 & 43.4 & 8.7 & 3.2 & 51 & 0 & 0.85 & 44 \\
\hline 14 & 46.7 & 3.5 & 2.6 & - & - & - & - \\
\hline 15 & 50.4 & 8.9 & 3.6 & 59 & 0.05 & 0.85 & 37 \\
\hline 16 & 53.9 & 4.7 & 2.7 & - & - & - & - \\
\hline 17 & 57.7 & 8.6 & 4.5 & 67 & 0 & 0.86 & 31 \\
\hline 18 & 61.8 & 8.9 & 3.5 & 72 & 0.04 & 0.86 & 30 \\
\hline I & 5.3 & - & - & $6-7$ & 0.3 & $0.76-0.88$ & $289-596$ \\
\hline II & 14.1 & - & - & 14 & 0.3 & 1.0 & 0 \\
\hline III & 21.9 & - & - & 23 & 0.3 & 0.95 & 28 \\
\hline
\end{tabular}

a transition from electron-shell to atomic-shell structure at a conductance $G \sim 40 G_{0}$. When the shallow peaks A-D are included (green stars), the transition to atomic-shell structure occurs already at $G \sim 20 G_{0}$. Conversely, when only the largest-weight peaks $(>5 \%)$ at high conductance are included (blue + symbols), the slope corresponding to electron-shell structure is seen to extend all the way up to the highest peak fit, $G \sim 60 G_{0}$. Note that at a temperature sufficient to display electron-shell effects, the weights of the peaks with $G<20 G_{0}$ may be suppressed due to thermally-induced breaking of the contact, as previously observed in alkali metals 2 . Figure 6 suggests that electronic- and atomic-shell effects compete in the regime $20 G_{0}<G<60 G_{0}$, with atomic-shell effects becoming increasingly important with increasing $G$. 


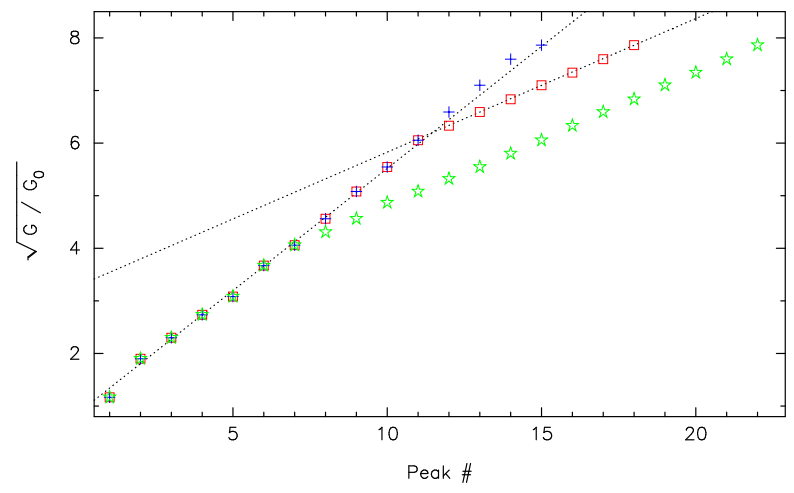

Figure 6. (colour online) Linear dependence of the peak positions in $\sqrt{G / G_{0}}$ using various selection criteria: (i) Main peaks, as in the inset of figure 2 (red squares); (ii) Keeping only the peaks with weight larger than $5 \%$ at large conductance (blue + symbols); (iii) Keeping all peaks in table 1 (green stars).

\subsection{Electronic Shell Effects}

Recently, the stability of alkali and noble metal nanowires was extensively studied theoretically [5, 6, 7, 17, 18, 27, 28, using the Nanoscale Free-Electron Model (NFEM) [8, 10. It was found that nanowire stability is determined by a competition between surface and shell contributions. A linear stability analysis considering cylindrical geometries as well as wires with broken axial symmetry revealed a "magic" sequence of stable nanowires, that allows for a consistent interpretation of experimental conductance histograms for alkali metals, including both the electronic shell and supershell structures 6, 17. The same sequence of stable wires is observed in histograms for $\mathrm{Au}$ 20, 21, suggesting a possible universality.

Guided by the importance of conduction electrons in the cohesion of metals, and by the success of the jellium model in describing metal clusters [11, 12, the NFEM replaces the metal ions by a uniform, positively charged background that provides a confining potential for the electrons. The electron motion is free along the wire, and confined in the transverse directions. The surface properties of various metals can be fit by using appropriate surface boundary conditions [29, 6].

Aluminum is a trivalent metal, but the Fermi surface of bulk $\mathrm{Al}$ resembles a freeelectron Fermi sphere in the extended-zone scheme. This suggests that the NFEM might be applicable to $\mathrm{Al}$ nanowires, although the continuum approximation is more severe than for monovalent metals. A first-principles theoretical calculation of the electronic structure of $\mathrm{Al}$ nanowires indeed reveals that, although the atomic structure has to be taken into account, the band structure has a free-electron like dispersion relation [30. Free-electron jellium models [12] have already successfully been used for modeling $\mathrm{Al}$ clusters [31, 32, 33] and explaining the occurrence of magic numbers [34, 35, 36. De Heer et al 31, 32] have specifically addressed the question of the applicability of the jellium model by comparing measured electronic properties of $\mathrm{Al}_{n}$ clusters to the values calculated with the jellium model. For the polarizability they find good agreement [31] for $n>40$, while when considering ionization potentials and electron affinities, it is found that clusters with $n>13$ are already accurately described by the jellium model 32 . Using a charge neutrality argument, the atomic 
volume is given by $v_{a}=9 \pi^{2} / k_{F}^{3}$ for a trivalent metal, the radius $R$ of an $\mathrm{Al}_{13}$ cluster can be estimated as $k_{F} R=6.5$, while that of $\mathrm{Al}_{40}$ is $k_{F} R=9.5$. Aluminum nanowires with these radii would have electrical conductances of $7 G_{0}$ and $18 G_{0}$, respectively, according to (1). We therefore expect the NFEM to become quantitatively accurate for nanowires with $G \gtrsim 12 G_{0}$.

Ogando et al [37] have self-consistently calculated the electron shell potential for Aluminum within a local density approximation using the stabilized jellium model. The free energy shows oscillations characteristic of the shell effect. Despite using a more elaborate self-consistent jellium model, their results predict the same sequence of energetically preferred cylindrical wires and the same positions for the supershell beat minima as the NFEM, which a posteriori underlines the applicability of our approach.

Guided by the points given above, we use the NFEM to interpret the electronshell structure observed in figure 2. A general method for performing linear stability analysis of metal nanowires, including the modelling of material-specific surface properties, was recently presented [18. Here, we apply the method of [18 to Aluminum and concentrate on presenting the results.

We examine straight wires aligned along the $z$-axis and, in order to account for an energetically favorable symmetry breaking (Jahn-Teller effect), both cylindrical and quadrupolar cross sections are considered. The wire geometry is given by the radius function

$$
r(\varphi)=\rho\left(\sqrt{1-\lambda^{2} / 2}+\lambda \cos (2 \varphi)\right)
$$

in cylindrical coordinates $r, \varphi, z$, characterized by the rms radius $\rho$ and the quadrupole deformation parameter $\lambda$. For a given geometry $(\rho, \lambda)$ we calculate the grand canonical potential $\Omega$ of the electron gas and examine its change with respect to small perturbations. Only if $\Omega$ increases for every possible small deformation of the initial geometry is the wire (linearly) stable. Note that this rigorous thermodynamic definition of stability is not the same as identifying stable wires as minima of the shell potential, as is done for example in 37. Although the most-stable wires do correspond to minima of the shell potential, not every minimum of the shell potential corresponds to a stable wire, as has been shown by considering non-axisymmetric deformations [18.

Figure 7 displays the results of the stability analysis as a function of temperature: shaded (coloured) areas represent stable geometries, where the deviation from axial symmetry $\lambda$ is coded via a gray (colour) scale. The temperature is displayed in units of $T_{\rho} \equiv T_{F} / k_{F} \rho$, where $T_{F}$ is the Fermi temperature, which provides a good measure of the total stability of a metal nanowire [18.

A series of very stable cylindrical wires can be identified, with conductance values $G / G_{0}=3,6,12,17,23,34,42,51, \ldots$ Their absolute stability is periodically modulated, and remarkably stable quadrupolar wires are found at the nodes of this supershell structure at $G / G_{0}=9,29,59, \ldots$ These two series combined form the "universal" series of stable wires explaining the observed shell and supershell structures in alkali and noble metals. Two additional stable highly-deformed wires that are well separated in conductance from their neighbors, and are thus expected to give pronounced peaks in the conductance histogram, are labeled peak Nos. 2 and 6 in figure 7 The theoretical quantized conductance values and quadrupole deformations of this sequence of stable nanowires are given in table 1 .

In figure 8 , this theoretical series of most stable wires is compared to the dominant (electron-shell) peaks extracted from the histogram of figure 2, plotted as (blue) 


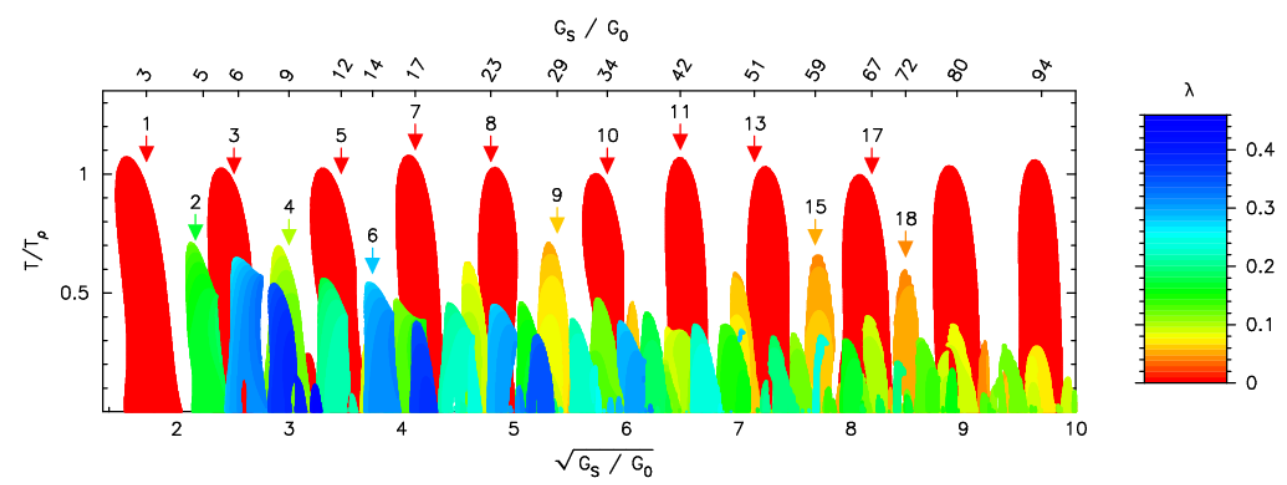

Figure 7. (colour online) Energetically stable cylindrical and quadrupolar Al wires as function of temperature (in units of $T_{\rho}=T_{F} / k_{F} \rho$, see text). The deviation from axial symmetry is coded via the gray (colour) scale shown on the right. The numbered arrows label the configurations that enter the analysis of the shell structure and give the number of the corresponding peaks in the histogram.

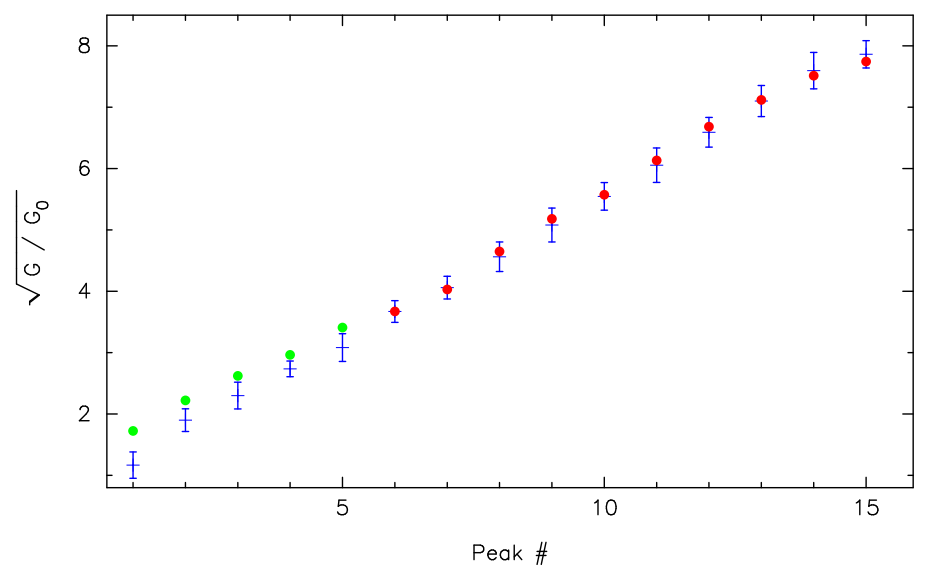

Figure 8. (colour online) Comparison of the stable $\mathrm{Al}$ nanowires within the NFEM (solid circles) with the dominant experimental peaks (blue symbols with error bars). Here the experimental error bars indicate the peak widths, and the theoretical conductance values were corrected by a mean series resistance of $36 \Omega$. The NFEM predictions for the low-conductance peaks are shown in green, for completeness.

+ symbols in figure 6. The theoretical conductance values have been corrected by subtracting a series resistance of $36 \Omega$, which was fitted to phenomenologically describe the backscattering of electron waves by defects, surface irregularities, and phonons [38, 39. Aside from a systematic shift in the theoretical peaks with $G \leq 12 G_{0}$, where the NFEM is expected to be inaccurate, there is a perfect one-to-one correspondence of the stable $\mathrm{Al}$ nanowires determined within the NFEM and the experimental electronshell structure. This correspondence is also shown in table 1, along with the mean channel transmission $\left\langle T_{n}\right\rangle$ (or series resistance $R_{s}$ ) necessary to shift the theoretical quantized conductance values down to the experimental values. As expected 24, $\left\langle T_{n}\right\rangle$ is significantly less than one for contacts with 3-6 channels due to strong mode 
mixing of the $s$ - and $p$-orbitals, but it approaches unity for $G>12 G_{0}$. Eventually, $\left\langle T_{n}\right\rangle$ is expected to decrease again as the system crosses over from ballistic to diffusive transport.

The NFEM predicts a rather universal sequence of dominant peaks in conductance histograms, stemming from electron-shell effects. This sequence is expected to be present for free-electron-like metals, and is consistent with previous experiments on alkali [2, 6] and noble metals [20, 21, and is confirmed by the data on Aluminum reported here. For non free-electron-like materials, other models must be used to determine the stability. For example, classical molecular dynamics simulations such as [40, predict very different, material specific (i.e. nonuniversal) low conductance histograms for $\mathrm{Ag}, \mathrm{Pt}$ and $\mathrm{Ni}$ nanowires.

\subsection{Atomic Shell Effects}

Similar to what is seen in aluminum clusters [1], the conductance histogram in figure 2 shows a crossover to a different peak period of about $\Delta \sqrt{G / G_{0}} \simeq 0.25$ at larger conductance values. This shift in periodicity is associated with the appearance of a series of peaks of increasing intensity (cf. peaks A, B, C, D, 12, 14, and 16 in figure 5) that cannot be explained in terms of electron shell effects. This new periodicity can be understood as an atomic shell effect: at large diameters the nanowire will be crystalline and stable wires are expected to form for densely-packed structures with closed facets, that minimize the surface energy. In the case of face-centered cubic lattice, the [110] orientation has been shown to be most favorable to form nicely faceted long nanowires [42, 43. We assume that the bulk fcc packing of aluminum is preserved in nanowires similar to what has been observed for gold and silver nanowires in transmission electron microscopy by Rodrigues et al. [42, 44]. The lowest energy surfaces for $\mathrm{Al}$ are the (111) planes [45] and we can construct a densely packed wire along a [110] axis with four (111) facets and completing the hexagon with two larger (100) facets. One obtains a nearly hexagonal cross-section of the wire illustrated in the inset of figure 1. The faceting structure of stable wires is reproduced in a Monte Carlo simulation of the thinning-down of a fcc wire by Jagla and Tosatti 43. Filling a whole atomic shell results in an increase of the nanowire radius with the period of $\Delta \sqrt{G / G_{0}}=1.98$. Assuming that stable wires are found when completing a single facet of the hexagon, one obtains a six times smaller period $\Delta \sqrt{G / G_{0}}=0.33$. An average experimental period obtained from 11 separate measurements would give an value of about $\Delta \sqrt{G / G_{0}} \sim 0.3$. As in the case of the electronic shell effect period, the agreement can be improved by correcting the theoretical value by subtracting a mean series resistance of $36 \Omega$.

On top of this correction, a lower period is expected due to the interplay between electronic and atomic shell effects. The relatively higher slopes observed for samples where the atomic shell effect is dominant, like in the histogram of figure1 as compared to the histogram of figure 2 comes in support to this assumption.

The crossover between electronic and atomic shell filling varies between different experiments, similar to what was found for alkali and noble metal nanowires [19, 21, 22] which is likely due to differences in the local crystal orientation of the leads connecting the nanowire. On top of this, for a given histogram the cross-over position is somewhat arbitrary, depending on the peak-selection criteria (see figure 6). When considering only the dominant peaks in the conductance histogram, mostly a value close to $40 G_{0}$ is observed. Still, the crossover from electronic to atomic shell effects is found to be 


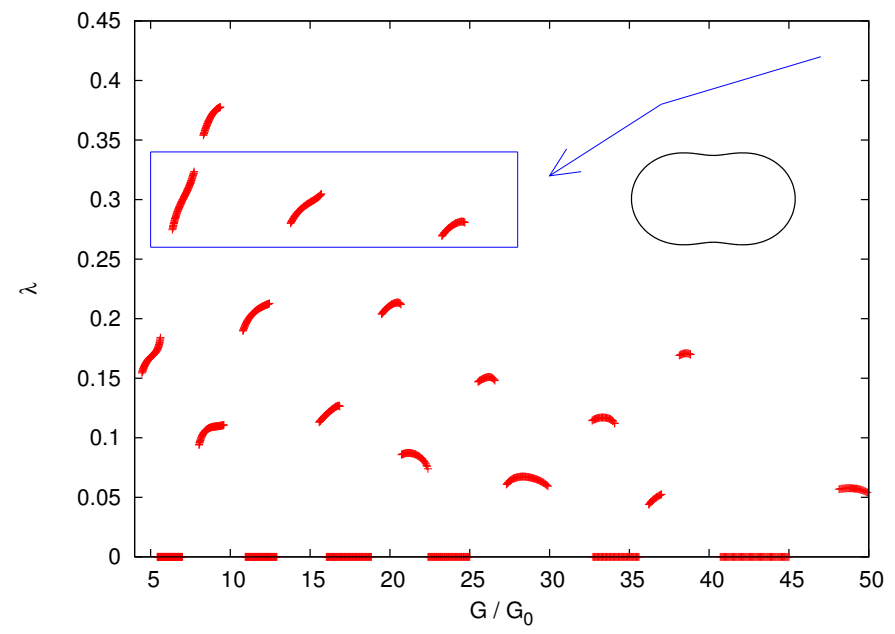

Figure 9. (colour online) Stability diagram for $\mathrm{Al}$ wires at fixed temperature $T=0.4 T_{\rho}$. Thick (red) lines mark stable wires in the configuration space of conductance $G$ and deformation parameter $\lambda$. The box emphasizes a series of very stable superdeformed wires, whose peanut-shaped cross section is shown as an inset. This sequence is thought to be responsible for the type of histogram shown in figure 3

a smooth transition rather than a sharp feature, if we include the number of shallow "peaks" (cf. peaks A, B, C, and D in figure 5) which start to show up at $G \simeq 19 G_{0}$ (cf. figure 6 and discussion).

These findings are in striking analogy to what was previously found for $\mathrm{Al}$ clusters. The early photoionization spectrometry measurements [34, 35, 36] on $\mathrm{Al}_{n}$ report electronic shell structure up to $n \sim 100$ while subsequent experiments [46, 47] give evidence for atomic shell effects for $n \gtrsim 200$. The crossover from electronic to atomic shell effects was studied in [41, 48] and a crossover in the range of $n \sim 75-100$ atoms was reported 41. We can convert the number of atoms to a radius as in section 3.1 using $v_{a}=9 \pi^{2} / k_{F}^{3}$. Then the crossover occurs in the range of $k_{F} \rho \simeq 11.7-12.8$, which corresponds to $G / G_{0} \simeq 28-35$ via (1), which agrees well with our observations.

\subsection{Superdeformed wires}

The third type of histogram seen in our experiment (cf. figure 3) is qualitatively different. It shows up only at the beginning of the measurement on a fresh sample, just after the initial breaking. Two dominant peaks near 14 and $22 G_{0}$ are observed, along with a third peak near $5 G_{0}$ in two out of seven cases. An intriguing possibility is that this third type of histogram is due to an isolated sequence of "superdeformed" nanowires, that is, nanowires whose cross section has an aspect ratio near 2:1. Superdeformation is a well-known feature of nuclear shell structure [49, but has not been previously observed in nanowires.

Our stability analysis shows that the series of wires with $\lambda \sim 0.3$ (aspect ratio $\approx$ 1.9 ), with conductances of 6,14 , and $23 G_{0}$, although less stable than the magic cylinders, are quite stable compared to other highly-deformed structures (cf. figure 7). More importantly, they are very isolated in configuration space, as illustrated in 
figure 9. Thus, if the initial structure of the nanocontact formed in the break junction is rather planar, with a large aspect ratio, then as the junction is pulled apart, it will maintain a large aspect ratio as it necks down elastically. Along such a trajectory in configuration space (schematically indicated by a blue arrow in figure 9), the most stable structures available are those of the superdeformed family (indicated by the blue box). Moreover, it is plausible that under elastic deformation during repeated elongation/compression cycles, the system would simply jump back and forth between these superdeformed structures, which are geometrically similar to each other. Eventually, however, a large structural fluctuation could drive the nanocontact into the axisymmetric lower energy minimum, where it would remain during further breaking cycles. This scenario is analogous to the decay-out process of highly-excited superdeformed nuclei, which remain in the superdeformed energy well thoughout a long sequence of decays, before finally tunnelling into the (lower minimum) normaldeformed energy well [49].

Evidence of superdeformation has not been reported in any of the previous experiments on alkali and noble metals, presumably because highly-deformed structures are intrinsically less stable than nearly axisymmetric structures, due to their larger surface energy. Aluminum is unique in this respect, since its surface tension is very small in natural units (i.e., in units of $E_{F} k_{F}^{2}$ ), some five times smaller than the value for gold [50], thus favoring the possibility of large deformations [18.

\section{Summary and Conclusions}

We report experimental and theoretical evidence that the stability of aluminum nanowires is governed by shell filling effects. The experimental investigation is done by recording conductance histograms at room temperature in UHV. Two "magic" series of stable structures are observed and the corresponding periodicities of the histogram peaks are very similar to the ones obtained recently for noble and alkali metal nanowires. Therefore we suggest, that also for $\mathrm{Al}$ the exceptionally stable structures are related to electronic and atomic shell effects. Concerning the electronic shell effect, the NFEM can explain the conductance and geometry (cylindrical or quadrupolar) of the stable structures. On the other hand, the atomic shell effect peaks can be understood considering close-packed hexagonal structures. In addition, new stable structures are found, that sometimes appear isolated in the conductance histogram. Following our stability analysis, we can attribute them to superdeformed nanowires. Remarkably, some of the peaks having the period expected for electronic shell effect survive under ambient conditions.

\section{Acknowledgments}

We would like to thank Gijs van Dorp for assistance with the measurements. This work is part of the research program of the "Stichting FOM" (A.I.M. and J.M.v.R) and it was supported by the EU Training Network DIENOW (A.I.M., D.F.U., H.G. and J.M.v.R), the DFG (D.F.U. and H.G.) and by NSF Grant Nos. 0312028 (J.B. and C.A.S.) and 0351964 (J.B.). 
Electronic and atomic shell structure in aluminum nanowires

\section{References}

[1] N. Agraï, A. Levy Yeyati, and J. M. van Ruitenbeek. Quantum properties of atomic-sized conductors. Phys. Rep., 377:81, 2003.

[2] A. I. Yanson, I. K. Yanson, and J. M. van Ruitenbeek. Observation of shell structure in sodium nanowires. Nature, 400:144, 1999.

[3] A. I. Yanson, I. K. Yanson, and Jan M. van Ruitenbeek. Shell effects in alkali metal nanowires. Fizika Nizkikh Temperatur, 27:1092, 2001.

[4] A. I. Yanson, I. K. Yanson, and J. M. van Ruitenbeek. Molecular Nanowires and other Quantum Objects, page 243. Kluwert Academic Publishers, Dordrecht, 2004.

[5] Frank Kassubek, Charles A. Stafford, Hermann Grabert, and Raymond E. Goldstein. Quantum suppression of the Rayleigh instability in nanowires. Nonlinearity, 14:167, 2001.

[6] D. F. Urban, J. Bürki, C.-H. Zhang, C. A. Stafford, and H. Grabert. Jahn-teller distortions and supershell effect in metal nanowires. Phys. Rev. Lett., 93:18640, 2004.

[7] J. Bürki, C. A. Stafford, and D. L. Stein. Theory of metastability in simple metal nanowires. Physical Review Letters, 95(9):090601, 2005.

[8] C. A. Stafford, D. Baeriswyl, and J. Bürki. Jellium model of metallic nanocohesion. Phys. Rev. Lett., 79:2863, 1997.

[9] J. M. van Ruitenbeek, M. H. Devoret, D. Esteve, and C. Urbina. Conductance quantization in metals: The influence of subband formation on the relative stability of specific contact diameters. Phys. Rev. B, 56:12566, 1997.

[10] J. Bürki and C. A. Stafford. On the stability and structural dynamics of metal nanowires. Applied Physics A, 81:1519, 2005.

[11] W. A. de Heer. The physics of simple metal clusters: experimental aspects and simple models. Rev. Mod. Phys., 65:611, 1993.

[12] M. Brack. The physics of simple metal clusters: self-consistent jellium model and semiclassical approaches. Rev. Mod. Phys., 65:677, 1993.

[13] T. P. Martin. Shells of atoms. Phys. Rep., 273:199, 1996.

[14] J. A. Torres, J. I. Pascual, and J. J. Sáenz. Theory of conduction through narrow constrictions in a three-dimensional electron gas. Phys. Rev. B, 49:16581, 1994.

[15] C. Höppler and W. Zwerger. Comment on jellium model of metallic nanocohesion. Phys. Rev. Lett., 80:1792, 1998.

[16] A. I. Yanson, I. K. Yanson, and J. M. van Ruitenbeek. Supershell structure in alkali metal nanowires. Phys. Rev. Lett., 84(25):5832-5835, 2000.

[17] D. F. Urban, J. Bürki, A. I. Yanson, I. K. Yanson, C. A. Stafford, J. M. van Ruitenbeek, and H. Grabert. Electronic shell effects and the stability of alkali nanowires. Solid State Com., 131:609, 2004.

[18] D. F. Urban, J. Bürki, C. A. Stafford, and H. Grabert. Stability and symmetry breaking in metal nanowires: The nanoscale free-electron model. Phys. Rev. B, 74:245414, 2006.

[19] A. I. Yanson, I. K. Yanson, and J. M. van Ruitenbeek. Crossover from electronic to atomic shell structure in alkali metal nanowires. Phys. Rev. Lett., 87:216805, 2001.

[20] M. Díaz, J. L. Costa-Krämer, E. Medina, A. Hasmy, and P. A. Serena. Evidence of shell structures in Au nanowires at room temperature. Nanotechnology, 14:113, 2003.

[21] A. I. Mares, A. F. Otte, L. G. Soukiassian, R. H. M. Smit, and J. M. van Ruitenbeek. Observation of electronic and atomic shell effects in gold nanowires. Phys. Rev. B, 70:073401, 2004.

[22] A. I. Mares and J. M. van Ruitenbeek. Observation of shell effects in nanowires for the noble metals $\mathrm{Cu}, \mathrm{Ag}$ and Au. Phys. Rev. B, 72:205402, 2005.

[23] A. I. Yanson and J. M. van Ruitenbeek. Do histograms constitute a proof for conductance quantization? Phys. Rev. Lett., 79:2157, 1997.

[24] E. Scheer, P. Joyez, D. Esteve, C. Urbina, and M. H. Devoret. Conduction channel transmissions of atomic-size aluminum contacts. Phys. Rev. Lett., 78:3535, 1997.

[25] E. Medina, M. Díaz, N. León, C. Guerrero, A. Hasmy, P. A. Serena, and J. L. Costa-Krämer. Ionic shell and subshell structures in aluminum and gold nanocontacts. Phys. Rev. Lett., 91:026802, 2003.

[26] K. Hansen, S. K. Nielsen, M. Brandbyge, E. Lægsgaard, I. Stensgaard, and F. Besenbacher. Current-voltage curves of gold quantum point contacts revisited. Appl. Phys. Lett., 77:708, 2000.

[27] Daniel F. Urban and Hermann Grabert. Interplay of Rayleigh and Peierls instabilities in metallic nanowires. Phys. Rev. Lett., 91:256803, 2003.

[28] J. Bürki, Raymond E. Goldstein, and C. A. Stafford. Quantum necking in stressed nanowires. Phys. Rev. Lett., 91(25):254501, 2003. 
[29] Antonio García-Martin, J. A. Torres, and J. J. Sáenz. Finite size corrections to the conductance of ballistic wires. Phys. Rev. B, 54(19):13448, 1996.

[30] F. Di Tolla, A. Dal Corso, J.A. Torres, and E. Tosatti. Electronic properties of ultra-thin aluminum nanowires. Surface Science, 454:947, 2000.

[31] W.A. de Heer, P. Milani, and A. Chatelain. Nonjellium-to-jellium transition in aluminum cluster polarizabilities. Phys. Rev. Lett., 63(26):2834, 1989.

[32] P. Milani, W. de Heer, and A. Châtelain. Electronic properties of aluminum clusters compared with the jellium model. Z. Phys. D, 19:133, 1991.

[33] M. F. Jarrold and J. E. Bower. Mobilities of metal cluster ions - aluminum and the electronic shell-model. J. Chem. Phys., 98(3):2399, 1993.

[34] K.E. Schriver, J.L. Persson, E.C. Honea, and R.L Whetten. Electronic shell structure of groupiiia metal atomic clusters. Phys. Rev. Lett., 64(21):2539, 1990.

[35] J. L. Persson, R. L. Whetten, H.-P. Cheng, and R. S. Berry. Evidence for quantized electronic level structure for 100-1300 electrons in metal-atomic clusters. Chem. Phys. Lett., 186:215, 1991.

[36] M. Pellarin, B. Baguenard, M. Broyer, J. Lerme, J.L. Vialle, and A. Perez. Shell structure in photoionization spectra of large aluminum clusters. J. Chem. Phys., 98(2):944, 1993.

[37] E. Ogando, N. Zabala, and M. J. Puska. Analysis of the shell- and supershell structures of metallic nanowires with jellium models. Nanotechnology, 13:363, 2002.

[38] B. Ludoph, M. H. Devoret, D. Esteve, C. Urbina, and J. M. van Ruitenbeek. Evidence for saturation of channel transmission from conductance fluctuations in atomic-size point contacts. Phys. Rev. Lett., 82(7):1530, 1999.

[39] J. Bürki, C. A. Stafford, X. Zotos, and D. Baeriswyl. Cohesion and conductance of disordered metallic point contacts. Phys. Rev. B, 60:5000, 1999.

[40] F. Pauly, M. Dreher, J. K. Viljas, M. Hafner, J. C. Cuevas, and P. Nielaba. Theoretical analysis of the conductance histograms and structural properties of $\mathrm{Ag}, \mathrm{Pt}$, and Ni nanocontacts. Phys. Rev. B, 74(23):235106, 2006.

[41] X. Li, H. Wu, X. B. Wang, and L. S. Wang. s-p hybridization and electron shell structures in Al clusters:a photolectron spectroscopy study. Phys. Rev. Lett., 81:1909, 1998.

[42] V. Rodrigues, T. Fuhrer, and D. Ugarte. Signature of atomic structure in the quantum conductance of gold nanowires. Phys. Rev. Lett., 85:4124, 2000.

[43] E. A. Jagla and E. Tosatti. Structure and evolution of a metallic nanowire-tip junction. Phys. Rev. B, 64:205412, 2001.

[44] V. Rodrigues, J. Bettini, A. R. Rocha, L. G. C. Rego, and D. Ugarte. Quantum conductance in silver nanowires: Correlation between atomic structure and transport properties. Phys. Rev. $B, 65: 153402,2002$

[45] L. Vitos, A.V. Ruban, H.L. Skriver, and J. Kollár. The surface energy of metals. Surf. Sci., 411:186, 1998.

[46] T. P. Martin, U. Naher, and H. Schaber. Evidence for octahedral shell structure in aluminum clusters. Chem. Phys. Lett., 199(5):470, 1992.

[47] U. Naher, U. Zimmermann, and T. P. Martin. Geometrical shell structure of clusters. J. Chem. Phys., 99(3):2256, 1993.

[48] B. Baguenard, M. Pellarin, J. Lerme, J. L. Vialle, and M. Broyer. Competition between atomic shell and electronic shell structures in aluminum clusters. J. Chem. Phys., 100(1):754, 1994.

[49] P. J. Nolan and P. J. Twin. Superdeformed shapes at high angular momentum. Annu. Rev. Nucl. Part. Sci., 38:533, 1988.

[50] W. R. Tyson and W. A. Miller. Surface free energies of solid metals: estimation from liquid surface tension measurements. Surf. Sci., 62:267, 1977. 